\section{Avaliação do impacto de programas de assistência pré-natal, parto e ao recém-nascido nas mortes neonatais evitáveis em Pernambuco, Brasil: estudo de adequação}

\author{
Assessment of the impact of prenatal, childbirth, \\ and neonatal care on avoidable neonatal deaths \\ in Pernambuco State, Brazil: an adequacy study \\ Evaluación del impacto de programas de asistencia \\ pre-natal, parto y cuidados al recién-nacido en \\ las muertes neonatales evitables en Pernambuco, \\ Brasil: estudio de adecuación
}

\section{Resumo}

Este estudo teve como objetivo avaliar o impacto de programas voltados à assistência pré-natal, parto e ao recém-nascido (Mãe Coruja Pernambucana e Rede Cegonha) na mortalidade neonatal evitável no Estado de Pernambuco, Brasil, utilizando a abordagem de adequação. Analisou-se a tendência dos coeficientes de mortalidade neonatal evitável, bem como o impacto desses programas na mortalidade neonatal evitável em quatro regiões de saúde do estado, de 2000 a 2016. Sistemas de Informações sobre Mortalidade (SIM) e de Nascidos Vivos (SINASC) e documentos oficiais foram usados como fonte de dados. Os óbitos foram classificados segundo a Lista Brasileira de Causas de Óbitos Evitáveis por Intervenções do SUS. Utilizaram-se métodos de regressão linear e joinpoint para análise das tendências e identificação de pontos de inflexão nas curvas de mortalidade neonatal. Houve acentuada queda da mortalidade neonatal evitável no estado, principalmente a precoce. Excetuando-se a Região I-Recife, onde observou-se inflexão negativa das curvas de mortalidade após a implantação da Rede Cegonha, não houve correspondência das inflexões nas curvas com os períodos de implantação dos programas nas demais regiões. Outros fatores parecem ter atuado na melhoria desses indicadores, como a ampliação da rede de alto risco. Portanto, o fortalecimento dessa rede pode contribuir na redução dos óbitos neonatais evitáveis, particularmente o precoce.

Planos e Programas de Saúde; Mortalidade Neonatal; Avaliação do Impacto na Saúde
Suzanne Santos de Lima 1

Maria Cynthia Braga 2

Lygia Carmen de Moraes Vanderlei 3

Carlos Feitosa Luna 2

Paulo Germano Frias 3

doi: 10.1590/0102-311X00039719

\author{
Correspondência \\ S. S. Lima \\ Rua José de Alencar 447, apto. 401, Recife, PE 50070-030, \\ Brasil. \\ suzanne-lima@hotmail.com \\ 1 Universidade de Pernambuco, Recife, Brasil. \\ 2 Instituto Aggeu Magalhães, Fundação Oswaldo Cruz, Recife, \\ Brasil. \\ 3 Instituto de Medicina Integral Prof. Fernando Figueira, Recife, \\ Brasil.
}




\section{Introdução}

Os avanços qualidade da assistência e o maior acesso às ações e serviços voltados à saúde materno-infantil, juntamente com a melhoria nas condições socioeconômicas da população, têm contribuído para o acentuado declínio da mortalidade infantil no mundo ${ }^{1}$. Todavia, um expressivo número de óbitos por causas evitáveis em menores de um ano, inclusive no período neonatal (de 0 a 27 dias de vida), persiste em países e regiões com menor nível de desenvolvimento 1,2 .

A partir da década de 1970, foram desenvolvidas propostas de classificação de mortes evitáveis para avaliar a qualidade da assistência à saúde, levando-se em consideração as diferentes dimensões envolvidas na determinação da mortalidade, como as relacionadas à atenção à saúde, às ações de saúde pública e às condições de vida 3,4,5. No Brasil, uma das classificações mais adotadas é a Lista de Causas de Mortes Evitáveis por Intervenções do Sistema Único de Saúde (SUS) 6, elaborada com base em revisão conceitual e empírica sobre mortes evitáveis e reflexões de um grupo de especialistas sob o ponto de vista do contexto nacional e recursos tecnológicos disponíveis no sistema. Com base nessa lista, constatou-se que a maioria das mortes neonatais evitáveis no país, notificadas no Departamento de Informática do SUS - DATASUS (http://tabnet.datasus.gov.br/cgi/ deftohtm.exe?sim/cnv/evita10uf. def, acessado em 17/Mar/2017) em 2014, foi classificada como causas relacionadas à atenção adequada durante a gestação (53,7\%), atenção ao recém-nascido (17\%) e ao parto $(9,8 \%)$.

Para o enfrentamento da morbimortalidade neonatal, diversas intervenções públicas foram direcionadas à saúde materno-infantil, da gestação ao cuidado com o recém-nascido no país 7 . No Estado de Pernambuco, além da adesão aos programas nacionais, foi lançado o Mãe Coruja Pernambucana, em 2007, que propõe reduzir a mortalidade infantil e materna nas regiões mais vulneráveis do estado por meio de ações intersetoriais e de saúde dirigidas às gestantes durante o pré-natal, parto e pós-parto e da criança, até o quinto ano de vida ${ }^{8}$. Posteriormente, o estado aderiu ao Rede Cegonha, instituído pelo Ministério da Saúde em 2011, que objetiva assegurar acesso, acolhimento e resolutividade ao parto, nascimento e à saúde da criança, com o protagonismo da mulher e a humanização da assistência 9 .

Apesar das evidências de que as ações propostas em ambos os programas tenham elevado potencial de reduzir a mortalidade neonatal 10,11, estudos avaliando a efetividade desses programas no Brasil não foram identificados em bases de dados indexadas.

Múltiplas abordagens metodológicas suscitam diferentes níveis de inferência na avaliação de impacto, como estudos de probabilidade, plausibilidade e de adequação 12. Os dois primeiros determinam se os resultados são decorrentes do programa avaliado, e o último mostra se as tendências dos indicadores de resultado estão na direção esperada 12. Diante da insuficiência de estudos avaliativos do Mãe Coruja e da Rede Cegonha no âmbito estadual, este trabalho teve como objetivo avaliar o impacto de programas voltados à assistência pré-natal, parto e ao recém-nascido na mortalidade neonatal evitável no Estado de Pernambuco, utilizando a abordagem de adequação.

\section{Métodos}

Trata-se de um estudo ecológico, de série temporal, usando dados dos Sistemas de Informações sobre Nascidos Vivos (SINASC) e sobre Mortalidade (SIM). O desenho tem um componente descritivo, para observação da tendência global dos coeficientes de mortalidade neonatal (CMN) evitáveis no estado; e um componente analítico, para análise das tendências dos CMN evitáveis, após o lançamento do Mãe Coruja Pernambucana e da Rede Cegonha, em quatro regiões de saúde selecionadas, por meio da avaliação de impacto com o nível de inferência de adequação 12. Essa abordagem metodológica de avaliação de impacto consiste na análise da tendência dos indicadores de resultados, antes e após a implantação do programa, verificando a direção e sua magnitude 12. Apesar de não permitir afirmar se os resultados são decorrentes exclusivamente da intervenção, essa abordagem possibilita uma avaliação inicial de impacto e levantamento de hipóteses para posterior aplicação de outros métodos 12 .

O Estado de Pernambuco, com população de 8.796.448 habitantes em 2010, é dividido em 12 regiões de saúde que são agrupadas em quatro macrorregiões: Metropolitana, Agreste, Sertão e Vale de São Francisco/Araripe. Para a análise de impacto, foram selecionadas quatro regiões de 
saúde representativas de cada uma dessas macrorregiões do estado: I-Recife, na Metropolitana; V-Garanhuns, no Agreste; IX-Ouricuri, no Vale de São Francisco/Araripe; e XI-Serra Talhada, no Sertão. A implantação dos programas Mãe Coruja Pernambucana e Rede Cegonha em todos os municípios também foi considerada como critério de escolha, exceto para a Região I-Recife, onde apenas o Município de Araçoiaba foi beneficiado pelo programa. Apesar disso, a Região I-Recife foi considerada na análise por concentrar o maior número de óbitos neonatais e de serviços de atenção ao recém-nascido de alto risco do estado.

As informações relativas aos programas (ano de implantação, ações previstas e executadas) foram obtidas por consulta a documentos institucionais disponibilizados pela Secretaria de Saúde de Pernambuco (SES-PE), apresentadas no Quadro 1. O Mãe Coruja cumpriu a maioria das ações previstas em todas as regiões estudadas, já o Rede Cegonha repassou recursos para a atenção básica, para a

\section{Quadro 1}

Ações previstas e implantadas pelos programas Mãe Coruja Pernambucana e Rede Cegonha, segundo Região de Saúde. Pernambuco, Brasil, $2007-2016$.

\begin{tabular}{|c|c|c|c|c|c|}
\hline \multirow{2}{*}{ Programas } & \multirow{2}{*}{ Ações previstas } & \multicolumn{4}{|c|}{ Execução das ações } \\
\hline & & I-Recife & V-Garanhuns & IX-Ouricuri & XI-Serra Talhada \\
\hline \multirow{10}{*}{$\begin{array}{l}\text { Mãe Coruja } \\
\text { Pernambucana * }\end{array}$} & $\begin{array}{l}\text { Implantação de canto Mãe Coruja nos } \\
\text { municípios }\end{array}$ & Parcial & Total & Total & Total \\
\hline & $\begin{array}{c}\text { Cadastro das gestantes e crianças }<5 \text { anos } \\
\text { usuárias do SUS }\end{array}$ & Parcial & Parcial & Parcial & Parcial \\
\hline & $\begin{array}{l}\text { Monitoramento de gestantes e crianças } \\
\text { cadastradas }\end{array}$ & Executada & Executada & Executada & Executada \\
\hline & Repasse de recursos a maternidades & Executada & Não prevista & Não prevista & Não prevista \\
\hline & $\begin{array}{l}\text { Equipagem com ultrassonógrafos em } \\
\text { maternidades }\end{array}$ & Executada & Não prevista & Não prevista & Não prevista \\
\hline & Estruturação dos cantos Mãe Coruja & Executada & Executada & Executada & Executada \\
\hline & Capacitação de profissionais & Executada & Executada & Executada & Executada \\
\hline & $\begin{array}{l}\text { Treinamento de profissionais dos cantos Mãe } \\
\text { Coruja }\end{array}$ & Executada & Executada & Executada & Executada \\
\hline & $\begin{array}{l}\text { Vigilância dos óbitos infantis nos cantos Mãe } \\
\qquad \text { Coruja }\end{array}$ & Executada & Executada & Executada & Executada \\
\hline & $\begin{array}{l}\text { Vinculação da gestante à rede de assistência } \\
\text { materno-infantil }\end{array}$ & Não executada & Não executada & Não executada & Não executada \\
\hline \multirow{8}{*}{ Rede Cegonha ** } & $\begin{array}{l}\text { Construção e equipagem de Centro de Parto } \\
\text { Normal }\end{array}$ & Não executada & Não executada & Não executada & Não executada \\
\hline & $\begin{array}{c}\text { Construção e equipagem de Casa da Gestante, } \\
\text { Bebê e Puérpera }\end{array}$ & Não executada & Não executada & Não executada & Não executada \\
\hline & $\begin{array}{l}\text { Investimentos para reforma e ampliação de } \\
\text { maternidades }\end{array}$ & Não executada & Não executada & Não executada & Não executada \\
\hline & Implantação e custeio de leitos de UTI neonatal & Parcial & Parcial & Parcial & Parcial \\
\hline & Implantação e custeio de leitos de UCI neonatal & Parcial & Parcial & Não prevista & Parcial \\
\hline & Custeio de UTI Canguru e Gestante de Alto Risco & Não executada & Não prevista & Não prevista & Não executada \\
\hline & Investimento em transporte sanitário & Não executada & Não executada & Não executada & Não executada \\
\hline & Repasse de recursos para a atenção básica & Executada & Executada & Executada & Executada \\
\hline
\end{tabular}

SUS: Sistema Único de Saúde; UCI: unidade de cuidados intermediários; UTI: unidade de tratamento intensivo.

* Ano de implantação da Mãe Coruja Pernambucana: 2008 (IX-Ouricuri) e 2009 (I-Recife, V-Garanhuns e XI-Serra Talhada);

** Ano de implantação da Rede Cegonha: 2012. 
implantação e custeio de leitos de UTI e UCI neonatais, e as demais ações não foram executadas como previsto.

Segundo dados do Ministério da Saúde, em 2016 todas as quatro regiões apresentavam cobertura de Estratégia Saúde da Família (ESF) acima de 85\%, exceto a Região I-Recife (59\%) (https://egestorab. saude.gov.br/paginas/acessoPublico/relatorios/relHistoricoCoberturaAB.xhtml, acessado em 14/ Jul/2019). Por outro lado, a Região I-Recife concentrou o maior número de médicos obstetras que atuavam no SUS (69\%), e foi a única região que apresentou número adequado destes profissionais (25 por 100 mil habitantes) (DATASUS. http://www2.datasus.gov.br/DATASUS/index.php?area=0204, acessado em 14/Jul/2019), segundo parâmetros oficiais 9 (Tabela 1). A quantidade de leitos obstétricos esteve acima dos parâmetros estabelecidos pelo Ministério da Saúde (0,28 por mil habitantes) em todas as regiões, exceto na I-Recife. Os leitos de UCI e UTI neonatais estavam concentrados na Região I-Recife, existindo vazios assistenciais nas demais, que dificultaram a análise do efeito da rede de alto risco sobre a mortalidade neonatal evitável nessas regiões. Assim, observa-se uma contração da rede de alta complexidade nas regiões mais afastadas da capital do estado, Recife (Tabela 1).

Considerando que o número de consultas de pré-natal indica o acesso à atenção de saúde durante a gestação, ainda que não mostre sua adequação, a tendência de cobertura pré-natal foi analisada, tal como os programas. Os dados sobre o número de consultas de pré-natal foram extraídos do SINASC, que apresenta proporção de dados ignorados ou sem informação desta variável de $0,9 \%$. O ponto de corte do número de sete consultas foi adotado com base na consulta de qualificação dos indicadores da Rede Interagencial de Informações para a Saúde (RIPSA) e para a padronização do dado, uma vez que nos anos estudados esta variável sofreu alterações na declaração de nascido vivo, passando de um campo com respostas categorizadas para um campo aberto 13,14 .

A população de estudo foi representada pelos nascidos vivos entre os anos de 1999 a 2016 e óbitos neonatais por causas evitáveis ocorridos de 2000 a 2016, residentes em Pernambuco e registrados no SINASC e SIM, respectivamente. Inicialmente, realizou-se a identificação e exclusão de registros duplicados do SINASC e SIM. Dos 2.654.141 nascidos vivos foram excluídos 2.611 (0,1\%), e dos 30.155 óbitos neonatais, 39 (0,1\%). Os óbitos neonatais e seus componentes precoce ( 0 a 6 dias de vida) e tardio (7 a 27 dias de vida) foram classificados de acordo com a causa básica com a Classificação Internacional de Doenças - 10a revisão (CID-10) 15 e segundo os critérios de evitabilidade, definidos na Lista Brasileira de Causas de Óbitos Evitáveis por Intervenções do SUS 6, para a obtenção dos coeficientes de mortalidade evitável (neonatal, neonatal precoce e tardia).

As análises de tendência temporal dos óbitos neonatais evitáveis e da cobertura pré-natal nas quatro regiões selecionadas foram conduzidas considerando-se o ano de implantação dos programas e o comportamento subsequente das séries temporais dos $\mathrm{CMN}$ evitáveis. $\mathrm{O}$ método de regressão joinpoint (https://surveillance.cancer.gov/joinpoint/) foi usado para a identificação de pontos de inflexão na curva de tendência dos coeficientes e para o cálculo dos percentuais de variação anual (PVA) do estado e das regiões selecionadas. Foram considerados estatisticamente significantes joinpoints com o valor de $\mathrm{p}<0,05$.

Tabela 1

Indicadores assistenciais da oferta de recursos humanos e leitos nas quatro regiões de saúde estudadas. Pernambuco, Brasil, $2005-2016$.

\begin{tabular}{|c|c|c|c|c|c|c|c|c|c|}
\hline \multirow[t]{3}{*}{ Indicadores } & \multirow[t]{3}{*}{ Parâmetro * } & \multicolumn{8}{|c|}{ Região de Saúde } \\
\hline & & \multicolumn{2}{|c|}{ I-Recife } & \multicolumn{2}{|c|}{ V-Garanhuns } & \multicolumn{2}{|c|}{ IX-Ouricuri } & \multicolumn{2}{|c|}{ XI-Serra Talhada } \\
\hline & & 2005 & 2016 & 2005 & 2016 & 2005 & 2016 & 2005 & 2016 \\
\hline Razão ginecologista-obstetra por 100 mil habitantes ** & 25 & 22,5 & 29,6 & 16,7 & 8,5 & 7,2 & 6,9 & 7,2 & 6,9 \\
\hline Leitos obstétrico por mil habitantes & 0,28 & 0,22 & 0,21 & 0,31 & 0,30 & 0,38 & 0,28 & 0,57 & 0,39 \\
\hline Leitos de UCI neonatal por mil nascidos vivos & 3 & 2,2 & 2,2 & 1,6 & - & - & - & - & - \\
\hline Leitos de UTI neonatal por mil nascidos vivos & 2 & 1,0 & 2,0 & - & - & - & - & - & - \\
\hline
\end{tabular}

ESF: Estratégia Saúde da Família; UCI: unidade de cuidados intermediários; UTI: unidade de tratamento intensivo.

Fonte: autora baseada no Cadastro Nacional de Estabelecimentos de Saúde (CNES), Instituto Brasileiro de Geografia e Estatística (IBGE) e Sistema de Informaçẽos sobre Nascidos Vivos (SINASC). 
O método de regressão linear simples foi aplicado para a análise da tendência dos coeficientes em todo o estado, uma vez que não foram observados pontos de inflexão na análise jointpoint. A adequação do modelo foi verificada pelos coeficientes de determinação $\left(R^{2}\right)$ e seus respectivos intervalos de $95 \%$ de confiança (IC95\%), e a velocidade e direção da tendência determinadas pelo valor de $\beta$. As curvas de tendência dos CMN evitáveis, CMNP evitável e CMNT evitável foram comparadas baseando-se na transformação dos dados das séries em variáveis dummy (variáveis binárias), binárias aditivas ou multiplicativas, por permitir verificar se duas equações lineares diferem em intercepto, em inclinação, ou ainda em ambos. As diferenças estatísticas entre as curvas de tendência foram testadas pelo teste $t$. Foram consideradas diferenças estatisticamente significantes valores de $\mathrm{p}<0,05$. As análises foram realizadas utilizando-se os programas SPSS, versão 20.0 (https://www.ibm.com/); Excel 2007 (https://products.office.com/) e Joinpoint Regress Program, versão 4.3.1.0 (https://surveillance.can cer.gov/joinpoint/).

O estudo foi aprovado pelo Comitê de Ética do Instituto Aggeu Magalhães, Fundação Oswaldo Cruz (CAEE56167116.5.0000.5190).

\section{Resultados}

De 2000 a 2016 foram registrados 2.482 .958 nascidos vivos e 49.737 óbitos em menores de um ano em Pernambuco, dos quais 30.119 (60,6\%) ocorreram no período neonatal e, destes, 68,1\% foram por causas evitáveis, a maioria $(79,9 \%)$ constituída por óbitos neonatais precoces.

Nesse período, os CMN evitáveis em Pernambuco declinaram de 13,3 para 6,6 óbitos/mil nascidos vivos, correspondendo a uma redução $50,4 \%(\beta=-0,402 ; \mathrm{p}=0,000)$. A análise da tendência pela regressão linear mostrou que a velocidade anual de queda do CMNP evitável, de 0,4 óbitos/mil nascidos vivos, foi significativamente maior $(\beta=-0,366 ; \mathrm{p}=0,000)$ do que a observada para o CMNT evitável ( 0,03 óbitos/mil nascidos vivos) ( $\beta=-0,037 ; p=0,009)$. Não houve diferença entre as inclinações dos CMN evitáveis e CMNP evitável $(t=1,446$; valor de $\mathrm{p}=0,155)$, porém houve diferença estatisticamente significante entre CMN evitáveis e CMNT evitável $(t=14,996$; valor de $\mathrm{p}<0,001)$ e entre CMNP evitável e CMNT evitável ( $\mathrm{t}=-13,550$; valor de $\mathrm{p}<0,001)$ (Figura 1).

A Tabela 1 e a Figura 2 mostram os resultados das análises de tendência dos CMNP e CMNT evitáveis e da cobertura de pré-natal, além dos pontos de inflexão nas séries temporais identificadas

\section{Figura 1}

Tendência do coeficiente de mortalidade neonatal, neonatal precoce e tardia por causas evitáveis, pelo método joinpoint e regressão linear. Pernambuco, Brasil, 2000-2016.

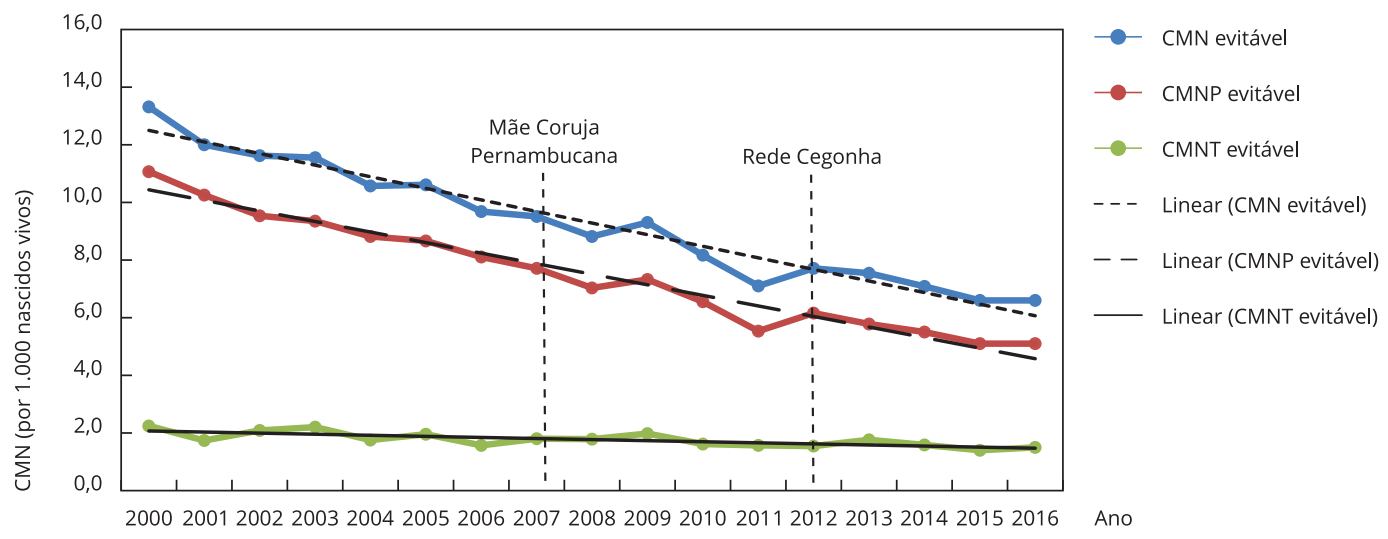


Figura 2

Tendência da mortalidade neonatal precoce e tardia evitável e cobertura de pré-natal nas regiões de saúde estudadas, pelo método joinpoint. Pernambuco, Brasil, 2000-2016.

2a) I-Recife
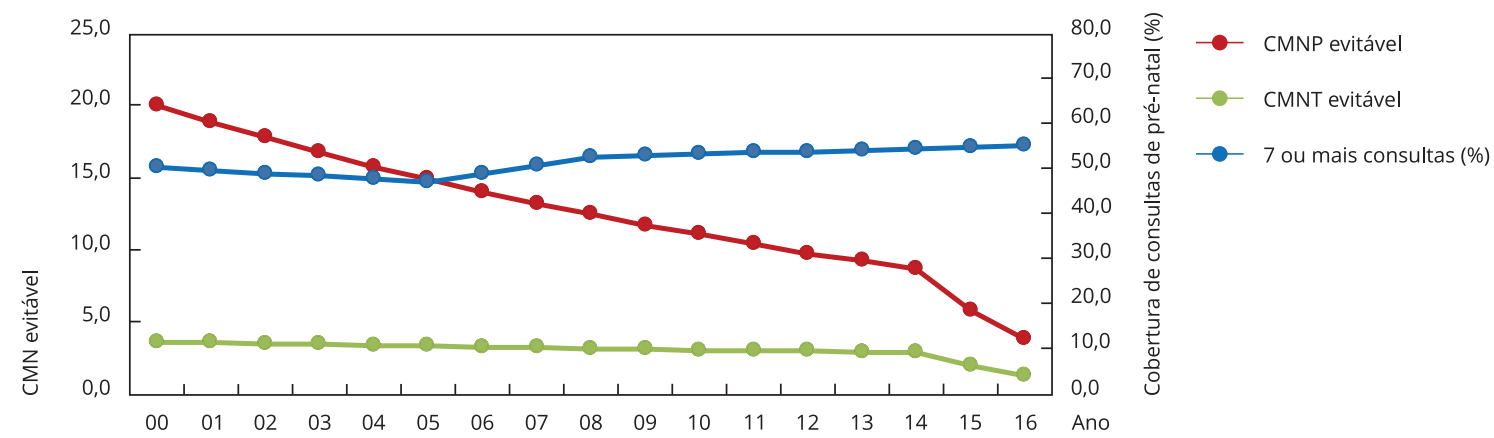

2b) V-Garanhuns
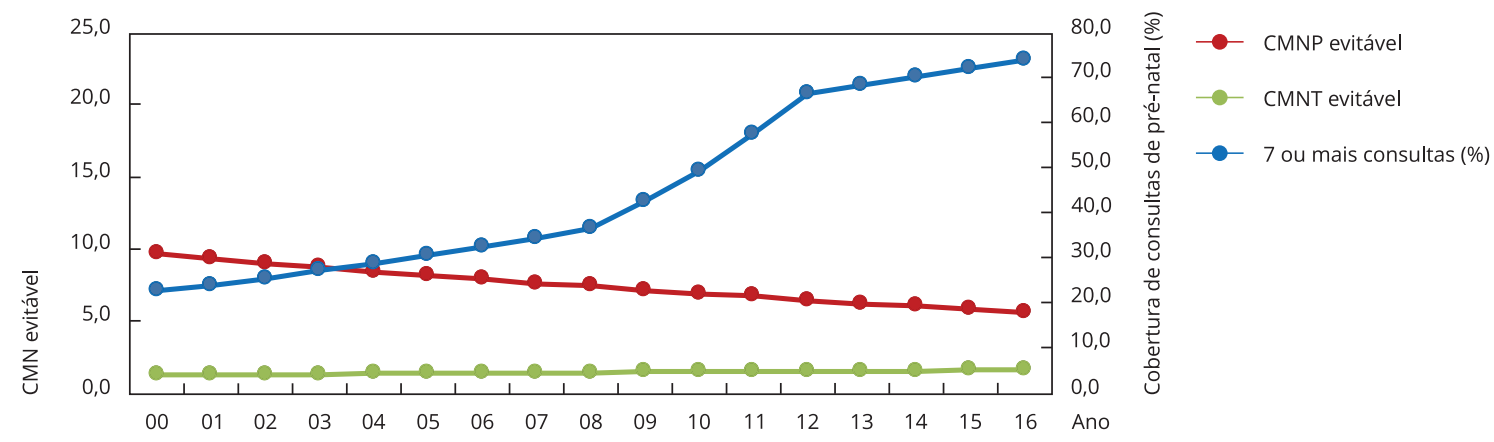

2c) IX-Ouricuri
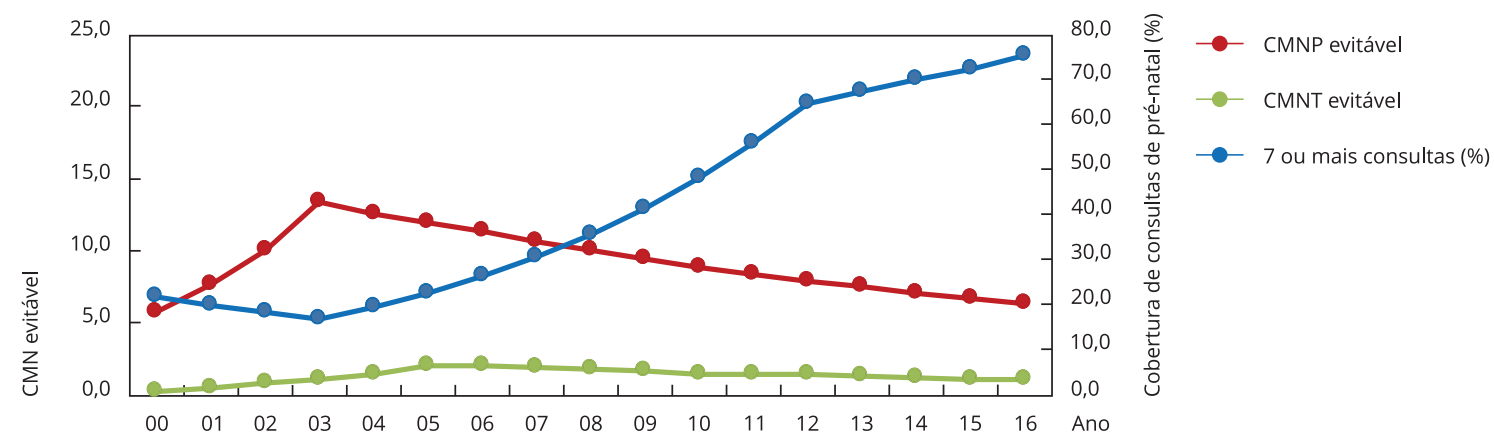


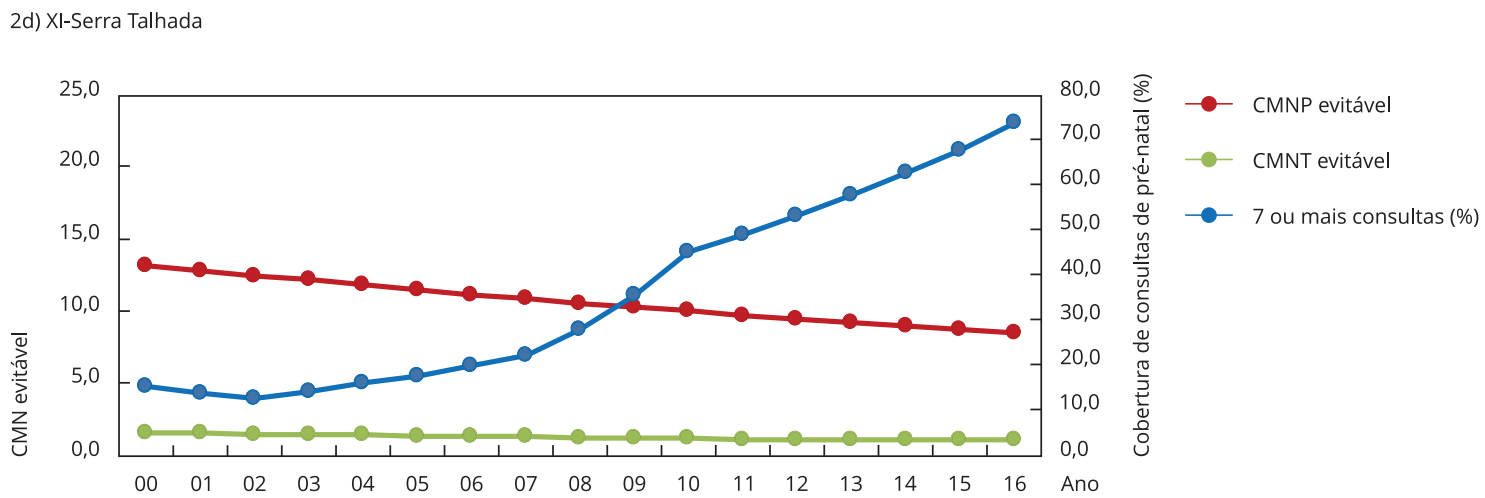

Nota: na Região de Saúde I-Recife o Mãe Coruja Pernambucana foi implantado apenas no Município de Araçoiaba.

pelo método joinpont, nas quatro regiões estudadas. Com relação ao CMNP evitável, ocorreu tendência de queda em todas as regiões, com exceção da IX-Ouricuri, onde houve tendência de aumento de 2000 a 2003 (PVA = 32,8; IC95\%: 7,3; 64,4) e, em seguida, de declínio entre 2003 e 2016 (PVA = -5,6; IC95\%: -7,7; -3,5), sendo este período anterior à implantação dos programas. Na Região I-Recife, o ponto de inflexão no CMNP evitável ocorreu imediatamente após a implantação da Rede Cegonha (PVA = -33,3; IC95\%: -51,0; -9,1), de 2014 a 2016. Nas regiões V-Garanhuns e XI-Serra Talhada houve suave tendência de queda do CMNP evitável ao longo de todo o período, sem pontos de inflexão.

Quanto ao CMNT evitável, observou-se ponto de inflexão negativo acentuado na curva de tendência da I-Recife (PVA = 32,1; IC95\%: -51,3; -5,2), no período de 2014 a 2016, e na IX-Ouricuri a inflexão foi positiva de 2000 a 2005 (PVA = 34,9; IC95\%: 3,6; 75,8). Nas regiões XI-Serra Talhada e V-Garanhuns não observou-se pontos de inflexão nas tendências (Tabela 2; Figura 2).

Com exceção da Região I-Recife, as demais, situadas no interior do estado, apresentaram pontos de inflexão estatisticamente significantes na curva de cobertura pré-natal. Na Região V-Garanhuns, ocorreu inflexão positiva entre 2000 e 2008 (PVA = 6,1; IC95\%: 4,1; 8,2), com acentuação à tendência entre 2008 a 2012 (PVA = 16,1; IC95\%: 6,4; 26). Na Região IX-Ouricuri, houve uma tendência de queda das coberturas entre 2000 e 2003 (PVA = -8,3; IC95\%: -14,8; -1,3), com posterior inflexão positiva de 2003 a 2012 (PVA = 16,0; IC95\%: 14,2; 17,9). Na Região XI-Serra Talhada, observou-se um ponto de inflexão positivo da curva no período entre 2002 e 2007 (PVA = 11,7; IC95\%: 5,6; 18,2), com intensificação da tendência positiva entre 2007 e 2010 (PVA = 26,4; IC95\%: 5,9; 50,9) e posterior desaceleração da inflexão positiva no período seguinte, de 2010 a 2016 (PVA = 8,5; IC95\%: 5,3; 11,8). A maior parte dos pontos de inflexão da cobertura de consultas de pré-natal ocorreu antes da implantação dos programas Mãe Coruja Pernambucana e Rede Cegonha.

\section{Discussão}

A análise mostrou que, embora o CMN evitável persista em patamares elevados, no Estado de Pernambuco e nas quatro regiões selecionadas houve redução acentuada destes coeficientes, particularmente o CMNP, não observando-se acentuação da tendência de redução com a implantação do programa Mãe Coruja Pernambucana e da Rede Cegonha, exceto na I-Recife, onde a redução foi expressiva. A acentuada tendência de ampliação da cobertura pré-natal nas regiões do interior não foi acompanhada pela correspondente redução do CMN evitável.

A maioria dos óbitos em menores um ano (mais de 60\%) ocorreu no período neonatal, principalmente na primeira semana de vida, majoritariamente por causas evitáveis, padrão similar ao ocorrido 


\section{Tabela 2}

Análise de tendência da mortalidade neonatal precoce e tardia evitável e cobertura de pré-natal nas quatro regiões de saúde estudadas, pelo método joinpoint. Pernambuco, Brasil, 2000-2016.

\begin{tabular}{|c|c|c|c|c|c|c|c|c|c|c|c|c|}
\hline \multirow{2}{*}{$\begin{array}{l}\text { Região de Saúde/ } \\
\text { Indicadores }\end{array}$} & \multicolumn{3}{|c|}{ Tendência 1} & \multicolumn{3}{|c|}{ Tendência 2} & \multicolumn{3}{|c|}{ Tendência 3} & \multicolumn{3}{|c|}{ Tendência 4} \\
\hline & Período & PVA & IC95\% & Período & PVA & IC95\% & Período & PVA & IC95\% & Período & PVA & IC95\% \\
\hline \multicolumn{13}{|l|}{ Pernambuco } \\
\hline CMNP evitável & $2000-2016$ & $-4,8$ & $-5,2 ;-4,4$ & & & & & & & & & \\
\hline CMNT evitável & $2000-2016$ & $-2,0$ & $-3,0 ;-1,0$ & & & & & & & & & \\
\hline $\begin{array}{l}\text { Cobertura de } \\
\text { consultas de } \\
\text { pré-natal }\end{array}$ & $2000-2005$ & 1,3 & 0,$3 ; 2,3$ & $2005-2016$ & 4,5 & 4,$2 ; 4,8$ & & & & & & \\
\hline \multicolumn{13}{|l|}{ I-Recife } \\
\hline CMNP evitável & $2000-2014$ & $-5,7$ & $-7,1 ;-4,4$ & 2014-2016 & $-33,3$ & $-51,0 ;-9,1$ & & & & & & \\
\hline CMNT evitável & $2000-2014$ & $-1,4$ & $-3,0 ; 0,1$ & 2014-2016 & $-32,1$ & $-51,3 ;-5,2$ & & & & & & \\
\hline $\begin{array}{l}\text { Cobertura de } \\
\text { consultas de } \\
\text { pré-natal }\end{array}$ & $2000-2005$ & $-1,3$ & $-2,6 ; 0,0$ & $2005-2008$ & 3,7 & $-2,4 ; 10,1$ & 2008-2016 & 0,6 & $-0,0 ; 1,3$ & & & \\
\hline \multicolumn{13}{|l|}{ V-Garanhuns } \\
\hline CMNP evitável & $2000-2016$ & $-3,3$ & $-4,6 ;-2,0$ & & & & & & & & & \\
\hline CMNT evitável & $2000-2016$ & 1,2 & $-1,8 ; 4,4$ & & & & & & & & & \\
\hline $\begin{array}{l}\text { Cobertura de } \\
\text { consultas de } \\
\text { pré-natal }\end{array}$ & $2000-2008$ & 6,1 & 4,$1 ; 8,2$ & 2008-2012 & 16,1 & 6,$4 ; 26,7$ & 2012-2016 & 2,6 & $-2,9 ; 8,5$ & & & \\
\hline \multicolumn{13}{|l|}{ IX-Ouricuri } \\
\hline CMNP evitável & $2000-2003$ & 32,8 & 7,$3 ; 64,4$ & 2003-2016 & $-5,6$ & $-7,7 ;-3,5$ & & & & & & \\
\hline CMNT evitável & $2000-2005$ & 34,9 & 3,$6 ; 75,8$ & $2005-2016$ & $-5,7$ & $-12,9 ; 2,1$ & & & & & & \\
\hline $\begin{array}{l}\text { Cobertura de } \\
\text { consultas de } \\
\text { pré-natal }\end{array}$ & $2000-2003$ & $-8,3$ & $-14,8 ;-1,3$ & $2003-2012$ & 16,0 & 14,$2 ; 17,9$ & $2012-2016$ & 3,8 & $-1,0 ; 8,7$ & & & \\
\hline XI-Serra & & & & & & & & & & & & \\
\hline CMNP evitável & 2000-2016 & $-2,7$ & $-4,0 ;-1,3$ & & & & & & & & & \\
\hline CMNT evitável & $2000-2016$ & $-2,4$ & $-7,6 ; 3,1$ & & & & & & & & & \\
\hline $\begin{array}{l}\text { Cobertura de } \\
\text { consultas de } \\
\text { pré-natal }\end{array}$ & $2000-2002$ & $-8,7$ & $-23,6 ; 8,9$ & 2002-2007 & 11,7 & 5,$6 ; 18,2$ & $2007-2010$ & 26,4 & 5,$9 ; 50,9$ & 2010-2016 & 8,5 & 5,$3 ; 11,8$ \\
\hline
\end{tabular}

CMN: coeficiente de mortalidade neonatal; CMNP: coeficiente de mortalidade neonatal precoce; CMNT: coeficiente de mortalidade neonatal tardio; IC95\%: intervalo de 95\% de confiança; PVA: percentual de variação anual.

há cerca de meio século nos países desenvolvidos 16 . No mundo, estimativas recentes publicadas em 2018 pelo Grupo Interagências das Nações Unidas para Estimação da Mortalidade Infantil (IGME) reportam que $47 \%$ dos óbitos em menores de cinco anos ocorreram no primeiro mês de vida, totalizando 2,5 milhões de mortes 17, a maioria nos países em desenvolvimento 10,18. Na América Latina e Caribe, para cada mil recém-nascidos, aproximadamente dez morrem no período neonatal 17.

Enquanto a redução dos óbitos pós-neonatais foi acentuada em decorrência da melhoria das condições de vida e à implantação de programas assistenciais 2,10,18,19, as mortes neonatais permanecem como desafio na maioria dos países, constituindo um dos 17 Objetivos de Desenvolvimento Sustentável (ODS) a serem alcançados até o ano de 2030 20. Internacionalmente, os óbitos neonatais evitáveis declinaram, entretanto, persistem grandes variações entre países e regiões, com reduções lentas e concentração de 75\% das mortes na África Subsaariana e no Sul da Ásia 21.

A análise mostrou que os CMN evitáveis apresentaram acentuada tendência de queda no Estado de Pernambuco, principalmente o CMNP evitável (0,4 óbitos/mil nascidos vivos) quando comparado 
ao CMNT (0,03 óbitos/mil nascidos vivos), entre 2000 e 2016, e no Brasil, na década de 2000, declinaram em 0,2 e 0,04 óbitos/mil nascidos vivos ao ano, respectivamente 6 . O menor declínio do CMNT provavelmente decorreu do fato de o coeficiente iniciar a série em patamar muito baixo.

O decréscimo das mortalidades materna, fetal e neonatal nos países de elevada renda sucedeu a uma gama de fatores, da dinâmica do sistema de saúde aos determinantes sociais, sinalizando que intervenções únicas raramente atingem reduções substanciais 16,22. A maioria das causas de mortes neonatais nos países de baixa renda está associada às condições maternas e fetais, entre elas, infecções, adquiridas durante a gestação e/ou parto; asfixia por complicações maternas, como descolamento da placenta ou pré-eclâmpsia; e a prematuridade, pela síndrome do desconforto respiratório, hemorragia intraventricular, enterocolitenecrosante e as infecções 16 . No Brasil, excetuando-se as malformações, o padrão predominante de mortes neonatais é evitável por ações oportunas e efetivas dos serviços de saúde, similar aos demais países de média e baixa rendas 19,23.

Em Pernambuco, apesar da redução dos coeficientes, a implantação dos programas Mãe Coruja Pernambucana e Rede Cegonha não acentuou a tendência de queda dos CMN evitáveis nas regiões de saúde situadas no interior do estado. Esse resultado foi diferente da Região I-Recife, onde verificou-se uma inflexão negativa das curvas de CMNP e CMNT evitável a partir de 2014, conforme evidenciado na análise joinpoint. Ao mesmo tempo, a análise mostrou que apesar da ampliação acentuada da cobertura pré-natal nas regiões do interior, com inflexões positivas, não foram observadas inflexões negativas dos CMN evitáveis ao longo da série. Ao contrário, na Região I-Recife houve inflexão negativa dos CMN evitáveis a partir de 2014, sem aumento expressivo da cobertura de pré-natal.

As intervenções necessárias à redução da mortalidade neonatal são bem estabelecidas e a sua oferta, pelo sistema de saúde, às mulheres e recém-nascidos, reduz acentuadamente o risco de desfechos perinatais desfavoráveis, desde que as coberturas sejam elevadas e de qualidade 14,21. O pré-natal adequado abrange ações de promoção à saúde, prevenção de doenças, detecção e tratamento precoce de complicações e preparação para o nascimento, efetivas na redução da mortalidade neonatal 16,19,23.

As boas práticas durante o trabalho de parto e parto também têm potencial de reduzir substancialmente o número de mortes neonatais 16,19 . Intervenções adequadas incluem a assistência por equipe multidisciplinar, que acompanhe a gestante desde a sua admissão no serviço, e utilização de protocolos que evitem procedimentos desnecessários 23 . A regionalização da assistência ao parto é outro fator que favorece o acesso a recursos tecnológicos e humanos de forma oportuna e adequada, cruciais para a sobrevivência neonatal, enquanto que a peregrinação da gestante em busca de vaga hospitalar aumenta em três vezes a chance de mortes neonatais 16,24,25. Por fim, cuidados adequados aos neonatos no pós-parto imediato, como a avaliação clínica eficiente, boas práticas de higiene, detecção precoce de infecção, ressuscitação neonatal, uso de surfactantes e/ou assistência ventilatória, suporte nutricional, além do incentivo à amamentação, dentre outros, têm papel relevante na redução da mortalidade neonatal 10,16,26.

No período de estudo, o Mãe Coruja Pernambucana executou as ações previstas, porém não priorizou as intervenções de maior impacto na mortalidade neonatal, como investimentos na rede de assistência de alta complexidade. Por outro lado, a Rede Cegonha, apesar de preconizar ações dirigidas aos determinantes da mortalidade, mediante a ampliação da rede por meio do aporte de recursos financeiros e de profissionais habilitados, pode não ter alcançado os resultados esperados nas regiões do interior do estado devido à sua implantação recente (2012) e a não ampliação da rede assistencial no período previsto.

A redução de mortalidade neonatal evitável com pontos de inflexão negativos se limitou à Região I-Recife, após a implantação da Rede Cegonha, provavelmente em decorrência da ampliação da rede de assistência, particularmente leitos de UTI neonatal, e de profissionais de saúde; já no interior do estado foram observados vazios assistenciais que podem ter repercutido nos indicadores. Nascimentos em hospitais que não dispõem de UTI neonatal aumentam em 2,5 vezes a chance de morte neonatal 19.

Existem evidências robustas sobre quais intervenções são necessárias e custo-efetivas na redução da mortalidade neonatal, grande parte delas disponível no Brasil, sem que sua cobertura seja adequada ou direcionada ao público que mais precisa, ou ainda utilizadas em tempo inoportuno com desempenho insuficiente 19,23,25,26. 
Uma das limitações do estudo está relacionada ao desenho utilizado, de avaliação de impacto com nível de inferência de adequação, por não permitir afirmar se a tendência do indicador esteve diretamente relacionada à intervenção, pois a análise adotada não permite o controle de outros fatores de confusão (distais, proximais) potencialmente relacionados à redução do CMN evitável. Para minimizar essa lacuna, usamos o método joinpoint, que permite identificar com relativa precisão, a magnitude, direção e inflexões no comportamento das curvas de mortalidade, após a implantação da intervenção, sugerindo seu efeito sobre o evento. Ressalta-se também que a cobertura, regularidade e qualidade dos dados melhoraram ao longo da série 27 , dado que fortalece os resultados da análise, considerando que tais fatores atuariam reduzindo a tendência de queda dos coeficientes.

O estudo mostrou forte redução dos CMN evitáveis, particularmente o precoce, não relacionado com a implantação dos programas estudados, sugerindo a influência de outros fatores na melhoria dos indicadores de mortalidade neonatal evitável. A exceção foi a influência positiva da Rede Cegonha na Região I-Recife, observada dois anos após a sua implantação, em particular o aumento da razão ginecologista-obstetra por 100 mil habitantes e a duplicação do número de leitos de UTI neonatal por mil nascidos vivos. Esses dados reforçam a importância do fortalecimento da rede de alta complexidade para a redução dos óbitos neonatais evitáveis, particularmente o precoce.

Para manter o avanço na redução da mortalidade neonatal evitável é necessário que as intervenções considerem os determinantes das mortes e o uso das melhores evidências científicas aplicáveis aos contextos locais. São igualmente importantes a realização de estudos com desenhos e análises que permitam estabelecer com maior precisão se os resultados foram decorrentes do programa avaliado.

\section{Colaboradores}

S. S. Lima, M. C. Braga, L. C. M. Vanderlei e P. G. Frias contribuíram com a concepção e desenho, aquisição de dados, análise e interpretação dos dados e elaboração do artigo. C. F. Luna contribuiu com a concepção e desenho, aquisição de dados, análise e interpretação dos dados e revisão crítica do conteúdo intelectual. Todos os autores aprovaram a versão a ser publicada e são responsáveis por todos os aspectos do trabalho.

\section{Informações adicionais}

ORCID: Suzanne Santos de Lima (0000-00030975-5306); Maria Cynthia Braga (0000-00027862-6455); Lygia Carmen de Moraes Vanderlei (0000-0002-3610-3699); Carlos Feitosa Luna (0000-0001-9277-4086); Paulo Germano Frias (0000-0003-4497-8898).

\section{Agradecimentos}

À Coordenação de Aperfeiçoamento de Pessoal de Nível Superior (Capes), pelo suporte para o desenvolvimento da pesquisa.

\section{Referências}

1. Wang H, Bhutta ZA, Coates MM, Coggeshall M, Dandona L, Diallo K, et al. Global, regional, national, and selected subnational levels of stillbirths, neonatal, infant, and under-5 mortality, 1980-2015: a systematic analysis for the Global Burden of Disease Study 2015. Lancet 2016; 388:1725-74.

2. Lehtonen L, Gimeno A, Parra-Lorca A, Vento M. Early neonatal death: a challenge worldwide. Semin Fetal Neonatal Med 2017; 22:15360.

3. Rutstein DD, Berenberg W, Chalmers TC, Child 3rd CG, Fishman AP, Perrin EB. Measuring the quality of medical care: a clinical method. N Engl J Med 1976; 294:582-8.

4. Taucher E. La mortalidad infantil en Chile. Notas Poblac 1979; 8:35-72.

5. Ortiz LP, Oushiro DA. Perfil da mortalidade neonatal no Estado de São Paulo. São Paulo Perspect 2008; 22:19-29.

6. Malta DC, Duarte EC, Almeida MD, Dias MAS, Morais Neto OL, Moura L, et al. Lista de causas de mortes evitáveis por intervenções do Sistema Único de Saúde do Brasil. Epidemiol Serv Saúde 2007; 16:233-44.

7. Araújo JP, Silva RMM, Collet N, Neves ET, Tos BRG, Viera CS. História da saúde da criança: conquistas, políticas e perspectivas. Rev Bras Enferm 2014; 67:1000-7. 
8. Secretaria Estadual de Saúde. Decreto no 30.859 , de 4 de outubro de 2007. Cria o Programa Mãe Coruja Pernambucana, e dá outras providências. Diário Oficial de Pernambuco 2007; 1 nov.

9. Ministério da Saúde. Portaria no 650 , de 5 de outubro de 2011. Diário Oficial da União 2011; 6 out.

10. Carlo WA, Travers CP. Mortalidade materna e neonatal: hora de agir. J Pediatr (Rio J.) 2016; 92:543-5.

11. Leal MC, Szwarcwald CL, Almeida PVB, Aquino EML, Barreto ML, Barros F, et al. Saúde reprodutiva, materna, neonatal e infantil nos 30 anos do Sistema Único de Saúde (SUS). Ciênc Saúde Colet 2018; 23:1915-28.

12. Habicht JP, Victora CG, Vaughan JP. Evaluation designs for adequacy, plausibility and probability of public health programme performance and impact. Int J Epidemiol 1999; 28:10-8.

13. Ministério da Saúde. Cobertura de consultas de pré-natal - F.6 - 2012. http://fichas.ripsa. org.br/2012/f-6/ (acessado em 14/Jul/2019).

14. Brasil. Lei no 12.662, de 5 de junho de 2012. Assegura validade nacional à Declaração de Nascido Vivo - DNV, regula sua expedição, altera a Lei no 6.015, de 31 de dezembro de 1973, e dá outras providências. Diário Oficial da União 2012; 6 jun.

15. Organização Mundial da Saúde. Classificação estatística internacional de doenças e problemas relacionados à saúde: CID-10. Décima revisão. 3a Ed. São Paulo: Edusp; 1996.

16. Goldenberg RL, McClure EM. Maternal, fetal and neonatal mortality: lessons learned from historical changes in high income countries and their potential application to low-income countries. Matern Health Neonatol Perinatol 2015; $1: 3$.

17. United Nations Inter-agency Group for Child Mortality Estimation. Levels \& trends in child mortality: report 2018. New York: United Nations Inter-agency Group for Child Mortality Estimation; 2018.

18. Liu L, Oza S, Hogan D, Perin J, Rudan I, Lawn JE, et al. Global, regional, and national causes of child mortality in 2000-13, with projections to inform post-2015 priorities: an updated systematic analysis. Lancet 2015; 385:430-40.
19. Lansky S, Friche AAL, Silva AAM, Campos D, Bittencourt SDA, Carvalho ML, et al. Pesquisa Nascer no Brasil: perfil da mortalidade neonatal e avaliação da assistência à gestante e ao recém-nascido. Cad Saúde Pública 2014; 30 Suppl 1:S192-207.

20. Programa das Nações Unidas para o Desenvolvimento. Objetivos de Desenvolvimento Sustentável. http://www.pnud.org.br/ods.aspx (acessado em 21/Mai/2016).

21. Akseer N, Lawnbj JE, Keenan W, Konstantopoulos A, Cooper P, Ismail Z, et al. Ending preventable newborn deaths in a generation. Int $\mathrm{J}$ Gynaecol Obstet 2015; 131:43-8.

22. Roos N, Xylander SR. Why do maternal and newborn deaths continue to occur? Best Pract Res Clin Obstet Gynaecol 2016; 36:30-44.

23. Bhutta ZA, Das JK, Bahl R, Lawn JE, Salam RA, Paul VK, et al. Can available interventions end preventable deaths in mothers, newborn babies, and stillbirths, and at what cost? Lancet 2014; 384:347-70.

24. Rodrigues NCP, Monteiro DLM, Almeida AS, Barros MBL, Pereira Neto A, O’Dwyer G, et al. Temporal and spatial evolution of maternal and neonatal mortality rates in Brazil, 19972012. J Pediatr (Rio J.) 2016; 92:567-73.

25. Leal MC, Theme-Filha MM, Moura EC, Cecatti JG, Santos LMP. Atenção ao pré-natal e parto em mulheres usuárias do sistema público de saúde residentes na Amazônia Legal e no Nordeste, Brasil 2010. Rev Bras Saúde Mater Infant 2015; 15:91-104.

26. World Health Organization. Every newborn: an action plan to end preventable deaths. http://www.healthynewbornnetwork.org/ hnn-content/uploads/Every_Newborn_Ac tion_Plan-ENGLISH_updated_July2014.pdf (acessado em 30/Mai/2018).

27. Frias PG, Pereira PMH, Andrade CLT, Lira PIC, Szwarcwald CL. Avaliação da adequação das informações de mortalidade e nascidos vivos no Estado de Pernambuco, Brasil. Cad Saúde Pública 2010; 26:671-81. 


\section{Abstract}

This study aimed to assess the impact of programs for prenatal, childbirth, and neonatal care (Mother Owl and Stork Network) on avoidable neonatal mortality in Pernambuco State, Brazil, using the adequacy approach. We analyzed the trend in avoidable neonatal mortality and the impact of these programs on avoidable neonatal mortality in four health regions in the state from 2000 to 2016. The Mortality Information System (SIM) and the Information System on Live Births (SINASC) and official documents were used as the data sources. Deaths were classified according to the Brazilian List of Avoidable Causes of Deaths Via Interventions by the Unified National Health System. Linear regression and joinpoint methods were used to analyze tendencies and identifying turning points in the neonatal mortality curves. There was a sharp drop in avoidable neonatal mortality in the state, especially in early neonatal mortality. Except for the I-Recife region, where there was a downturn in the mortality curves after implementation of the Stork Network, there was no association between the turning points in the curves and the periods with the programs' implementation in the regions. Other factors appear to have led to the improvement of these indicators, such as the expansion of the high-risk network. Strengthening this network can thus help reduce avoidable neonatal deaths, especially early deaths.

Health Programs and Plans; Neonatal Mortality; Health Impact Assessment

\section{Resumen}

El objetivo de este estudio fue evaluar el impacto de programas dirigidos a la asistencia pre-natal, parto y cuidados al recién nacido (Madre-Búho y Red Cigüeña) en la mortalidad neonatal evitable en el estado de Pernambuco, Brasil, utilizando un abordaje de adecuación. Se analizó la tendencia de los coeficientes de mortalidad neonatal evitable, así como el impacto de estos programas en la mortalidad neonatal evitable en cuatro regiones de salud del estado, de 2000 a 2016. Se utilizaron como fuente de datos los Sistemas de Información sobre Mortalidad (SIM) y de Nacidos Vivos (SINASC), así como documentos oficiales. Los óbitos se clasificaron según la Lista Brasileña de Causas de Óbitos Evitables por Intervenciones del SUS. Se utilizaron métodos de regresión lineal y joinpoint para el análisis de las tendencias e identificación de puntos de inflexión en la curvas de mortalidad neonatal. Hubo una acentuada caída de la mortalidad neonatal evitable en el estado, principalmente la precoz. Exceptuándose la región I-Recife, donde se observó una inflexión negativa de las curvas de mortalidad tras la implantación de la Red Cigüeña, no hubo una correspondencia de las inflexiones en las curvas con los periodos de implantación de los programas en las demás regiones. Otros factores parecen haber actuado en la mejoría de estos indicadores, como la ampliación de la red de alto riesgo. Por tanto, el fortalecimiento de esta red puede contribuir a la reducción de los óbitos neonatales evitables, particularmente el precoz.

Planes y Programas de Salud; Mortalidad Neonatal; Evaluación del Impacto en la Salud
Recebido em 28/Fev/2019

Versão final reapresentada em 24/Jul/2019 Aprovado em 14/Ago/2019 